\title{
The Wavelet Collocation Method Based on Economic Class of Equations Shannon
}

\author{
XIAO- HONG Dong ${ }^{1}$ a \\ ${ }^{1}$ School of Finance, Harbin University of Commerce, Harbin, China \\ aemail:wary_876@163.com
}

\begin{abstract}
Keywords: 2-D Shannon scale function. bivar tensor product. wavelet collocation method. The Economic Class of differential equations
\end{abstract}

Abstract. In view of the Economic Class of two-dimensional partial differential equation, a two dimensional scale function was chosen and was applied to the wavelet collocation method. Simultaneously, the two dimensional scaling basis function was proved satisfy the interpolate property. And according to two dimensional multi-resolution analysis theory, the approximate formulas of solutions for the Economic Class of the two dimensional differential equations were given. The two dimensional partial differential equations were separated by the wavelet collocation method and the system of ordinary differential equations was built. At last, the system of partial differential equations was solved. The result was better than the traditional Galerkin method and with higher precision.

\section{2-D Shannon wavelet collocation method}

Supposed the monadic function $\phi(x)$ to produce multi-resolution analysis, but the monadic function $\phi(y)$ produces another multi-resolution analysis, then $V_{j}^{1}$ and $V_{j}^{2}$ tensor product accumulates the space with the $V_{j}=V_{j}^{1} \otimes V_{j}^{2}$ Because the basis of $V_{j}^{1}$ was $\left\{\phi\left(2^{-j} x-k\right)\right\}$, but the basis of $V_{j}^{2}$ is $\left\{\phi\left(2^{-j} y-l\right)\right\}$, so the basis of $V_{j}$ was $\phi\left(2^{-j} x-k\right) \cdot \phi\left(2^{-j} y-l\right)$.The bivar function $f(x, y)$ may be introduced the symbol $f_{j, k, l}(x, y)=f\left(2^{-j} x-k, 2^{-j} y-l\right)$ Recording $\phi(x, y)=\phi(x) \cdot \phi(y)$ so $\left\{\phi_{j, k, l}(x, y) ; k, l \in Z\right\}$ was the basic of $V_{j}$ Then $\left\{V_{j}\right\}$ forms a multi-resolution analys was in $L^{2}(R \times R)$. But $\phi(x, y)$ was the corresponding scale function, take the Shannon scale function as the example, namely $\phi(x, y)=\phi(x) \cdot \phi(y)=\frac{\sin \pi x}{\pi x} \cdot \frac{\sin \pi y}{\pi y}$ The Figure 1. was produced by the tensor product of the two-dimensional Shannon scale function.

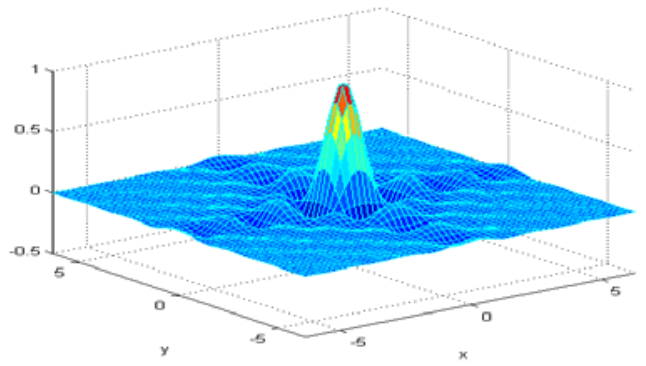

Fig1. 2-D Shannon scale function

\footnotetext{
${ }^{1}$ National Social Science Fund project (11BJY080) 、 (14BJL032) and National Science Foundation project (71340022)、Heilongjiang Province philosophy of social science projects (13B024) and Heilongjiang Provincial Department of Education Project (12541190).
} 


\subsection{The structure of basis function and its characteristic}

Considering the two-dimensional function $f(x, y)$, and taking the two-dimensional Shannon scale function to as the basis function, Because the situation of evenly discrete was more convenient than the non- even discrete. Therefore according to the multi-resolution analysis theory to the function $f(x, y)$ in its definition domain $[0, l] \times[0, l]{ }_{(l>0)}$ to carry on the even discrete., and the unit grid size records for $\Delta=\frac{l}{2^{j}} \quad$ ( $j$ was suitable integer),

$$
\begin{aligned}
& x_{m}=m \Delta, \quad m=0,1, \cdots, 2^{j} \\
& y_{n}=n \Delta, \quad n=0,1, \cdots, 2^{j}
\end{aligned}
$$

Definition basis function was

$$
w_{j, m, n}(x, y)=\frac{\sin \frac{\pi}{\Delta}\left(x-x_{m}\right)}{\frac{\pi}{\Delta}\left(x-x_{m}\right)} \cdot \frac{\sin \frac{\pi}{\Delta}\left(y-y_{n}\right)}{\frac{\pi}{\Delta}\left(y-y_{n}\right)}
$$

Meanwhile

$$
w_{j}\left(x-x_{k}\right)=\frac{\sin \frac{\pi}{\Delta}\left(x-x_{k}\right)}{\frac{\pi}{\Delta}\left(x-x_{k}\right)}
$$

Theory 1.1. The basis function was satisfied the following properties:

1. Interpolation $w_{j}\left(x_{i}-x_{k}\right)=\delta_{i k}$

2. Orthogonality $\int_{-\infty}^{+\infty} w_{j}\left(x-x_{i}\right) w_{j}\left(x-x_{k}\right) d x=\Delta \delta_{i k}$

3. Regeneration For any positive integer $n$ satisfing

$$
\int_{-\infty}^{+\infty} w_{j}\left(x-x_{i}\right) \frac{d^{n} w_{j}\left(x-x_{k}\right)}{d x^{n}} d x=\Delta \frac{d^{n} w_{j}\left(x_{i}-x_{k}\right)}{d x^{n}}
$$

Proof Here interpolation Clearly, we only proof orthogonality and Regeneration. The Fourier transform of basis function $w_{j}\left(x-x_{i}\right)$ was

$$
\hat{w}_{j}(a)=\left\{\begin{array}{lc}
\Delta & -\frac{\pi}{\Delta} \leq a \leq \frac{\pi}{\Delta} \\
0 & \text { others }
\end{array}\right.
$$

Parseval identical equationconcludes

$$
\begin{aligned}
& \int_{-\infty}^{+\infty} w_{j}\left(x-x_{i}\right) w_{j}\left(x-x_{k}\right) d x=\frac{1}{2 \pi} \int_{-\infty}^{+\infty} \hat{w}_{j}(\omega) e^{i x_{i} \omega} \hat{w}_{j}(\omega) e^{-i x_{k} \omega} d \omega \\
& =\frac{\Delta}{2 \pi} \int_{-\frac{\pi}{\Delta}}^{\frac{\pi}{\Delta}} \Delta e^{i \omega\left(x_{i}-x_{k}\right)} d \omega=\Delta w_{j}\left(x_{i}-x_{k}\right)
\end{aligned}
$$

Using interpolation concludes $\int_{-\infty}^{+\infty} w_{j}\left(x-x_{i}\right) w_{j}\left(x-x_{k}\right) d x=\Delta \sigma_{i k}$ Similarly, Parseval identical equation was concluded

$$
\begin{aligned}
& \int_{-\infty}^{+\infty} w_{j}\left(x-x_{i}\right) \frac{d^{n} w_{j}\left(x-x_{k}\right)}{d x^{n}} d x=\frac{1}{2 \pi} \int_{-\infty}^{+\infty} \hat{w}_{j}(\omega) e^{i x_{i} \omega} \hat{w}_{j}(\omega) e^{-i x_{k} \omega}(i \omega)^{n} d \omega \\
& =\frac{\Delta}{2 \pi} \int_{-\frac{\pi}{\Delta}}^{\frac{\pi}{\Delta}} \Delta e^{i \omega\left(x_{i}-x_{k}\right)}(i \omega)^{n} d \omega=\Delta \frac{d^{n} w_{j}\left(x_{i}-x_{k}\right)}{d x^{n}}
\end{aligned}
$$

Theory1.2. the basis function $w_{j_{, m, n}}\left(x_{m_{1}}, y_{n_{1}}\right)$ was satisfied the interpolation nature ,namely $w_{j, m, n}\left(x_{m_{1}}, y_{n_{1}}\right)=\delta_{m, m_{1}, n, n_{1}}$ 
Proof

$$
\begin{aligned}
w_{j, m, n}\left(x_{m_{1}}, y_{n_{1}}\right)= & \frac{\sin \frac{\pi}{\Delta}\left(x_{m_{1}}-x_{m}\right)}{\frac{\pi}{\Delta}\left(x_{m_{1}}-x_{m}\right)} \frac{\sin \frac{\pi}{\Delta}\left(y_{n_{1}}-y_{n}\right)}{\frac{\pi}{\Delta}\left(y_{n_{1}}-y_{n}\right)} \\
& = \begin{cases}1 & m_{1}=m \text { and } n_{1}=n \\
0 & m_{1} \neq m \text { or } n_{1} \neq n\end{cases} \\
& =\delta_{m, m_{1}, n, n_{1}}
\end{aligned}
$$

\subsection{The discrete forms of two-dimensional partial differential equations}

The Economic Class of the two-dimensional partial differential equation

$$
\begin{aligned}
& L(u)=f(x, y) \\
& A \frac{\partial^{2} u}{\partial x^{2}}+2 B \frac{\partial^{2} u}{\partial x \partial y}+C \frac{\partial^{2} u}{\partial y^{2}}+D \frac{\partial u}{\partial x}+E \frac{\partial u}{\partial y}+F u=f(x, y)
\end{aligned}
$$

Theory1.3 the approximate solution of $u(x, y)$ is $u_{j}(x, y) \in V_{j}(x, y)$ and may express is

$$
u_{j}(x, y)=\sum_{n_{1}=0}^{2^{j}} \sum_{n_{2}=0}^{2^{j}} u_{j}\left(x_{n_{1}}, y_{n_{2}}\right) w_{j, n_{1}, n_{2}}(x, y)
$$

and $u_{j}(x, y)$ converges $u(x, y)$.

the theory 1.2. was spreaded in where has published in Harbin University of Science andTechnology journal which was published in 11th volume 1st issue the center article the two-dimensional situation promotion, its proof was omitted. Making use of formula (2) was used wavelet collocation method to obtain its discrete form to formula (1),namely

$$
\begin{aligned}
& \sum_{n_{1}=0}^{2^{j}} \sum_{n_{2}=0}^{2^{j}} u_{j}\left(x_{n_{1}}, y_{n_{2}}\right)\left[A\left(x_{k_{1}}, y_{k_{2}}\right) \frac{\partial^{2} w_{j, n_{1}, n_{2}}\left(x_{k_{2}}, y_{k_{2}}\right)}{\partial x^{2}}+2 B\left(x_{k_{1}}, y_{k_{2}}\right) \frac{\partial^{2} w_{j, n_{1}, n_{2}}\left(x_{k_{1}}, y_{k_{2}}\right)}{\partial x \partial y}+C\left(x_{k_{1}}, y_{k_{2}}\right) \frac{\partial^{2} w_{j, n_{1}, n_{2}}\left(x_{k_{1}}, y_{k_{2}}\right)}{\partial y^{2}}\right. \\
& \left.+D\left(x_{k_{1}}, y_{k_{2}}\right) \frac{\partial w_{j, n, n_{2}}\left(x_{k_{1}}, y_{k_{2}}\right)}{\partial x}+E\left(x_{k_{1}}, y_{k_{2}}\right) \frac{\partial w_{j, n_{1}, n_{2}}\left(x_{k_{1}}, y_{k_{2}}\right)}{\partial y}+F\left(x_{k_{1}}, y_{k_{2}}\right) w_{j, n_{1}, n_{2}}\left(x_{k_{1}}, y_{k_{2}}\right)\right]=f\left(x_{k_{1}}, y_{k_{2}}\right) \\
& k_{1}=0,1,2, \cdots, 2^{j}, k_{2}=0,1,2, \cdots, 2^{j} .
\end{aligned}
$$

If the system of equations were solved, then we may obtain the approximate solution of two-dimensional partial differential equation (1) may be obtained.

\subsection{Numerical computation of the example}

Considering the two-dimensional ellipse partial differential equation.

$$
\left\{\begin{aligned}
-\Delta u=f & (x, y) \in \Omega=(0,1) \times(0,1) \\
u=0 & (x, y) \in \partial \Omega
\end{aligned}\right.
$$

including $f(x, y)=8 \pi^{2} \sin (2 \pi x) \sin (2 \pi y)$ Its analytic solution was $u(x, y)=\sin (2 \pi x) \sin (2 \pi y)$

Its Figure of analytic solution was Fig2.

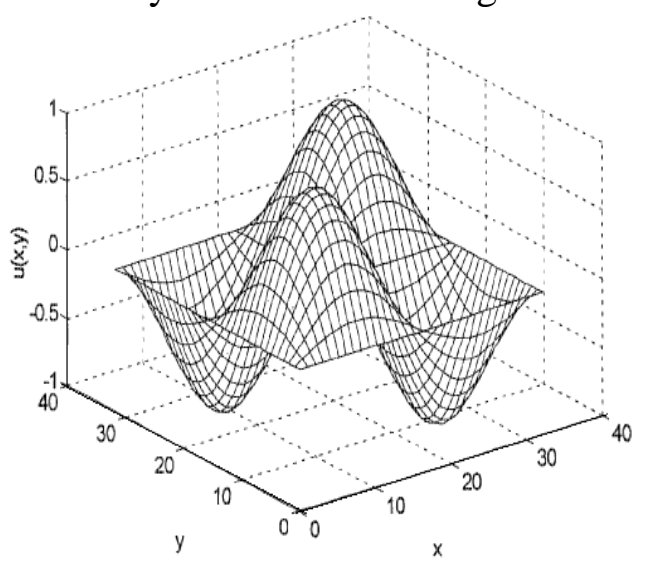

Figure2

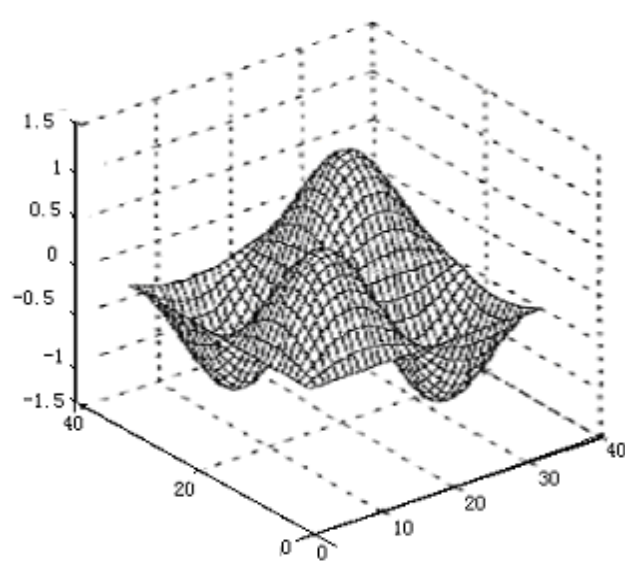

Figure3 
Figure2. analytic solution of wavelet collocation method when $j=6$ Figure3. was the numerical solution of wavelet collocation method when $j=6$.Figure 3 . the numerical solution of wavelet collocation method when $j=6$ Figure 4 . was the numerical solution of traditional Galerkin method.

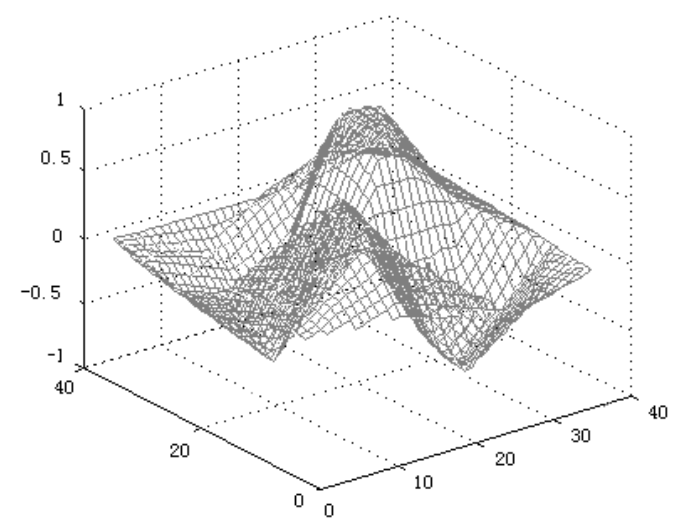

Fig 4. the numerical solution of traditional Galerkin method

Through the computed result chart, the two-dimensional wavelet collocation method obviously very well, Although it has the certain error with the analytic solution, the result is better than the traditional Galerkin method, moreover,it is more convenient and suitable.

\section{Conclusions}

Using the two-dimensional Shannon wavelet collocation method to discrete the Economic Class of two-dimensional partial differential equation, a group ordinary differential equation were succinctly obtained, thus will be reduced the difficulty to the partial differential equation.the result was better than the traditional Galerkin method .Obviously the resultwas satisfied.

\section{References}

[1] He Jihuan:Homotopy perturbation method : a new nonlinear analytical technique .Applied Mathematics and Computation, Vol. 26(2003),p.73 - 79

[2] Wei G W: Quasi wavelets and quasi interpolating wavelets.Chemical Phys ics Letters.Vol. 26(1998), ,p.215 -222

[3] Gehring:Wavelets and Differential Equations.Introduction to Wavelets Through Linear Algebra. Vol. 12(1999),p.451-483

[4] Daubechies:TheWavelet Transform. Time-frequency Localization and Signal Analysis. IEEE Trans. on Information Theory. Vol. 36(1990),p. 961-100

[5] Information on http://www.wavelet.gov.cn 